\title{
Suplementación de cromo sobre concentraciones séricas de glucosa, cortisol e insulina en equinos deportistas
}

\author{
Chromium suplementation on glucose, cortisol and insulin blood concentrations in sport horses
}

A Suplementação de cromo nas concentrações de glicose no sangue, cortisol e insulina em cavalos de esporte

\author{
Fernando Pazmiño Galarza \\ fapazmino@uce.edu.ec \\ https://orcid.org/0000-0003-2471-2048
}

Jorge Grijalva Olmedo
jgrijalva@uce.edu.ec
https://orcid.org/0000-0001-8301-531X

\author{
Lenin Romero Celleri \\ leninvet@hotmail.com \\ https://orcid.org/0000-0002-7056-6371
}

\author{
Eduardo Aragón Vázquez \\ earagon@uce.edu.ec \\ https://orcid.org/0000-0002-5142-0721
}

Universidad Central del Ecuador, Facultad de Medicina Veterinaria y Zootecnia, Ecuador

\section{RESUMEN}

El objetivo del estudio fue determinar como la suplementación de cromo afecta el rendimiento de caballos deportivos, se evaluó los posibles efectos de dos fuentes de suplementos de cromo orgánico en caballos atléticos. Se evaluaron 15 equinos deportivos, raza Argentina Saddle, de 12 a 15 años, alimentados con concentrado, pasto de centeno y heno de alfalfa. Fueron divididos en tres grupos experimentales: i) el grupo de control recibió dieta basal sin suplementación, ii) dieta basal suplementada con $20 \mathrm{mg}$ de cromo en forma de levadura enriquecida por 30 días; y iii) dieta basal suplementada con $20 \mathrm{mg}$ de cromo en forma de tripicolinato de cromo por 30 días. Después, se realizo una prueba submáxima de esfuerzo y posterior una evaluación de niveles sanguíneos de glucosa, cortisol e insulina. El análisis estadístico del nivel de glucosa no mostró diferencias significativas para ninguno de los tratamientos $(P>0.05)$, pre y post ejercicio. El cortisol plasmático fue similar entre el tratamiento pre-ejercicio, la levadura enriquecida con cromo y el cromo orgánico, disminuyeron el nivel de cortisol en sangre post ejercicio $(P<0,05)$. La concentración plasmática de insulina se vio influenciada por la suplementación con cromo después del ejercicio intensivo, los resultados demostraron que el nivel de insulina en sangre fue mayor en los caballos que no recibieron suplementación $(P<0.01)$ en comparación con los grupos que recibieron suplementación de cromo. La suplementación con cromo orgánico en caballos deportivos no modificó la glucosa plasmática, pero si la concentración sanguínea de cortisol e insulina.

Palabras clave: cromo; cortisol; glucosa; insulina; tripicolinato
ABSTRACT

The objective of the study was to determine how chromium supplementation affects the performance of sports horses, for which the possible effects of two sources of organic chromium supplements in athletic horses were evaluated. Fifteen sporting horses were evaluated, Argentina Saddle breed, aged 12 to 15 years, which were fed with concentrate, rye grass and alfalfa hay. They were divided into three experimental groups: i) the control group received a basal diet without supplementation, ii) a basal diet supplemented with $20 \mathrm{mg}$ of chromium in the form of enriched yeast for 30 days; and iii) basal diet supplemented with $20 \mathrm{mg}$ of chromium in the form of chromium tripicolinate for 30 days. After this time, a submaximal stress test was carried out and subsequent evaluation of blood levels of glucose, cortisol and insulin. The statistical analysis of the glucose level did not show significant differences for any of the treatments $(P>0.05)$, before and after exercise. Plasma cortisol was similar between pre-exercise treatment, but chromium-enriched yeast and organic chromium decreased post-exercise blood cortisol level $(P<0.05)$. Plasma insulin concentration was influenced by chromium supplementation after intensive exercise, the results showed that the blood insulin level was higher in the horses that did not receive supplementation $(P<0.01)$ compared to the groups that received supplementation. Chrome. Organic chromium supplementation in sporting horses did not modify plasma glucose, but the blood concentration of cortisol and insulin did.

Key words: Chromium; cortisol; glucose; insulin; tripicolinate

\section{RESUMO}

O objetivo do estudo foi determinar como a suplementação de cromo afeta o desempenho de cavalos esportivos, para os quais foram avaliados os possíveis efeitos de duas fontes de suplementos de cromo orgânico em cavalos atletas. Foram avaliados 15 cavalos esportivos da raça Argentina Saddle, com idades entre 12 e 15 anos, que foram alimentados com concentrado, centeio e feno de alfafa. Eles foram divididos em três grupos experimentais: i) o grupo controle recebeu dieta basal sem suplementação; ii) dieta basal suplementada com $20 \mathrm{mg}$ de cromo na forma de levedura enriquecido por 30 dias; e iii) Dieta basal suplementada com 20 $\mathrm{mg}$ de cromo como tripicolinato de cromo por 30 dias. Após esse tempo, foi realizado um teste de esforço submáximo e posterior avaliação dos níveis sanguíneos de glicose, cortisol e insulina. A análise estatística do nível de glicose não mostrou diferenças significativas para nenhum dos tratamentos $(P>0,05)$, antes e após o exercício. O cortisol plasmático foi semelhante entre o tratamento pré-exercício, mas a levedura enriquecida com cromo e o cromo orgânico diminuíram o nível de cortisol no sangue pós-exercício $(P<0,05)$. A concentração de insulina plasmática foi influenciada pela suplementação de cromo após exercícios intensivos, os resultados mostraram que o nível de insulina no sangue foi maior nos cavalos que não receberam suplementação $(P<0,01)$ em comparação com os grupos que receberam suplementação. A suplementação de cromo orgânico em cavalos de esporte não modificou a glicose plasmática, mas sim a concentração sangüínea de cortisol e insulina.

Palavras-Chave: cromo; cortisol; glicose; insulina; tripicolinato 


\section{INTRODUCCIÓN}

Los micro minerales son requeridos en pequeñas cantidades por los caballos, lo que juega un papel importante en todos los sistemas bioquímicos que influyen en todas las funciones metabólicas del animal $(1,2)$. El cromo $(\mathrm{Cr})$ es un micro mineral de transición involucrado en muchos eventos oxidativos y ha sido reconocido como un nutriente esencial en humanos $y$ animales. El cromo es un componente del factor de tolerancia a la glucosa (GTF), que maximiza la actividad de la insulina (3-4), especialmente en los tejidos con deficiencia de $\mathrm{Cr}$ (5). Por lo tanto, el $\mathrm{Cr}$ participa en el metabolismo de los carbohidratos y depende del proceso de la insulina, como el metabolismo de las proteínas y los lípidos (6-8).

Los requerimientos de cromo aumentan con la actividad física y, por lo tanto, es un mineral esencial para los caballos deportivos $(9,10,11)$. Los caballos deportivos normalmente se encuentran bajo estrés como resultado de la intensa actividad del ejercicio, su concentración de cortisol aumenta para mantener la glucosa en sangre en niveles más altos durante el ejercicio $(9,12)$. La glucosa es una fuente de energía esencial, por lo que una adecuada síntesis y uso es esencial para los caballos durante las carreras (13). Se demuestra que el $\mathrm{Cr}$ estimula el transporte de glucosa al interior de la célula, la síntesis de aminoácidos y previene la lipasa tisular (14) mediante una potenciación directa de la actividad de la insulina, que regula la captación de glucosa por parte de las células maximizando la actividad muscular proporcionando suficiente energía para el ejercicio prolongado (15).

Se ha descrito que la deficiencia de $\mathrm{Cr}$ puede incrementar las concentraciones séricas de colesterol y triacilglicerol y disminuir la concentración de insulina unida debido a la reducción del número de receptores de insulina. La suplementación con cromo orgánico se ha relacionado con la mejora de la respuesta inmunitaria, la disminución del nivel plasmático de cortisol en animales estresados y ayuda a la acumulación de proteínas en los músculos $(9,16)$. Además, estudios recientes demostraron los beneficios del cromo orgánico en equinos que padecen síndrome metabólico (SME) caracterizado por obesidad y resistencia a la insulina, los cuales han sido descritos como factores de riesgo para el desarrollo de laminitis $(13,15,17)$. Además, sugieren un mejor aprovechamiento de la fuente de energía mediante la suplementación con cromo, lo que contribuye a la disminución del lactato después del ejercicio (18).

Sin embargo, el Consejo Nacional de Investigación (NRC) no ha incluido el $\mathrm{Cr}$ como parte de los requerimientos de nutrientes para caballos, especialmente aquellos que están bajo ejercicio intensivo. Además, algunas preguntas sobre la suplementación micro mineral siguen sin respuesta, por lo que este artículo proporcionará una impresión sobre el efecto de diferentes fuentes de cromo orgánico sobre la concentración sanguínea de glucosa, cortisol e insulina en caballos sometidos a un entrenamiento intensivo de ejercicio.

\section{MATERIALES Y MÉTODOS}

El trabajo de campo se realizó en Sangolquí, Ecuador. Un total de quince machos castrados de la raza Caballo Argentino fueron utilizados en este diseño de estudio completamente al azar; El estudio se realizó de acuerdo con los estándares éticos del Comité de Ética de la Universidad Central del Ecuador y Centro Ecuestre del Ejército del Ecuador. Todos los caballos tenían entre 12 y 15 años y tenían al menos 2 años de entrenamiento intensivo. Los caballos se mantuvieron en establos $(3,5 \mathrm{~m} x$ $3,5 \mathrm{~m}$ ) en las mismas condiciones durante todo el experimento desde las 16:00 hasta las 10:00 am del día siguiente. Los caballos se dividieron 
en tres grupos y se asignaron al azar a los tratamientos. Los tratamientos consistieron en un grupo de control (dieta sin cromo) y dietas con dos fuentes diferentes de cromo: levadura de cromo (200 mg) y tripicolinato de cromo (200 mg). Las fuentes de cromo se incluyeron en la dieta normal de los caballos, que consistía en una mezcla de concentrado y forraje. Formulación de concentrado está en la lista en la Tabla 1, las raciones se formularon de acuerdo con las recomendaciones del NRC para los caballos adultos bajo actividad física intensiva (NRC, 2007). La mezcla de forraje consistió en pasto de centeno (Lolium perenne) y alfalfa (Medicago sativa) en una proporción de 3: 1. La proporción de ración de concentrado y forraje fue de 80:20. Los caballos fueron alimentados de acuerdo con su peso corporal (inicialmente a un nivel de 2,25\%) dos veces al día, 6:00 y 16:00 h durante dos semanas consecutivas.

Tabla 1. Formulación de concentrado.

\begin{tabular}{|c|c|}
\hline Ingredients & $\%$ \\
\hline Avena molida & 32.0 \\
\hline Maiz molido & 25.0 \\
\hline Harinilla de trigo & 10.7 \\
\hline Harina de soja (46\%) & 12.0 \\
\hline Salvado de trigo & 10.0 \\
\hline Aceite de soya & 3.0 \\
\hline Carbonato de soya & 1.3 \\
\hline Fosfato dicálcico & 1.0 \\
\hline Sal & 1.0 \\
\hline Melaza & 3.0 \\
\hline Propionato de calcio & 0.1 \\
\hline Premezcla Mineral-Vitamina & 1.0 \\
\hline
\end{tabular}

En esta tabla se encuentra la fórmula del concentrado administrada a los caballos deportistas basada en las recomendaciones del Consejo Nacional de Investigación.

Todos los caballos, después de haber sido alimentados con suplementos de cromo, se ejercitaron en una cinta de correr de baja y alta velocidad. Se evaluaron tres etapas de ejercicio: 1) etapa de paso (1000 ma 2,4 m s-1 velocidad promedio), 2) etapa de trote (1400 ma 3,8 m s-1 velocidad promedio) y 3 ) etapa de galope (1,000 a $9,5 \mathrm{~m} \mathrm{~s}-1$ velocidad media). Entrenamiento de caballos desde las 10:00 hasta completar el metro de la cinta. Una vez finalizada la prueba de ejercicio, los caballos se colocaron en el potrero diario de kikuyo (Pennisetum clandestinum).

Para este experimento, se tomaron cuatro muestras de sangre de la vena yugular de los caballos. Se recolectaron $5 \mathrm{~mL}$ de sangre por muestra y se colocaron en tubos vacutainer sin anticoagulante. Se tomó una muestra de sangre antes de todo el proceso de ejercicio (7:00), cuyos valores se consideraron como valores de control. 
Se tomaron tres muestras de sangre después del ejercicio, una inmediatamente después de terminar todas las etapas del ejercicio (tiempo $0)$ y dos más a las 3 y 6 horas posteriores a las etapas del ejercicio. Los tubos Vacutainer se centrifugaron a 10.000 rpm durante 5 minutos para obtener suero. Estas muestras se utilizaron para estimar la concentración sanguínea de glucosa, cortisol e insulina mediante ensayo enzimático colorimétrico e inmunoensayos enzimáticos, respectivamente.

Todos los datos recopilados en este estudio se ingresaron en Microsoft Excel 2011 y se analizaron en busca de errores y errores tipográficos. Las diferencias entre los tratamientos (fuente de $\mathrm{Cr}$ ) y el efecto del ejercicio sobre la concentración sanguínea de cortisol, glucosa e insulina se evaluaron mediante ANOVA en las puntuaciones medias. La probabilidad de significación se fijó en $\mathrm{P}$ $<0,05$. El análisis estadístico se realizó mediante el programa SPSS Statistics para Windows.

\section{RESULTADOS}

Los resultados obtenidos tras evaluar los niveles en sangre se observan en la Figura 1 , que muestra la variación de la media de concentración sanguínea de glucosa, cortisol e insulina por tratamientos $y$, antes y después de la exposición al ejercicio. En general, los parámetros sanguíneos de glucosa, cortisol e insulina difieren entre tratamientos, y su concentración era más alta antes de que los caballos realizaran una actividad física intensiva.

Por un lado, la concentración de glucosa varía entre tratamientos, siendo mayor en el grupo control (Figura 1a). Además, las concentraciones séricas de glucosa tienden a disminuir mientras aumenta el tiempo de post-ejercicio. El tratamiento con levadura $\mathrm{Cr}$ parece inducir una reducción progresiva de la concentración de glucosa en sangre, en comparación con la fuente de suplementación con Tripicolinato $\mathrm{Cr}$. Sin embargo, la diferencia de los niveles séricos de glucosa no fue significativa $(P>0.05)$ entre los tratamientos y el tiempo posterior al ejercicio. Por lo tanto, el efecto de las fuentes de $\mathrm{Cr}$ y la actividad física sobre el nivel sérico medio de glucosa fue similar en este estudio (Tabla 2).

Por otro lado, la concentración sérica de cortisol también es mayor en el grupo control, sin embargo, su concentración disminuye en el momento del post-ejercicio aumenta al igual que los otros tratamientos (Figura 1b). El tratamiento con levadura $\mathrm{Cr}$ indujo una reducción progresiva de la concentración de cortisol en sangre en comparación con el Tripicolinato $\mathrm{Cr}$. Sin embargo, la diferencia entre los tratamientos fue significativa sólo inmediatamente después de una intensa actividad de ejercicio $(P<0,05)$. Las fuentes de levadura $\mathrm{Cr}$ y tripicolinato $\mathrm{Cr}$ inducen un efecto similar sobre la concentración sérica de cortisol, que es una disminución (Tabla 3).

En el caso de la concentración de insulina en sangre, el comportamiento fue similar entre tratamientos, teniendo los mismos patrones en el momento del aumento post-ejercicio (Figura 1c). Sin embargo, el nivel de insulina fue mayor en los caballos alimentados con suplementos de Tripicolinato $\mathrm{Cr}$ que en el grupo de control y en el grupo de tripicolinato Cr. Además, la concentración de insulina fue muy cercana entre tratamientos inmediatamente después de la exposición al ejercicio (tiempo 0). Las dos fuentes de cromo producen un efecto similar sobre la concentración de insulina en sangre, sin embargo, se observaron diferencias significativas $(P<0.05)$ entre los tratamientos inmediatamente después del ejercicio y después de 3 horas de actividad de ejercicio intensivo (Tabla 4). 
Tabla 2. Media de la concentración de glucosa en sangre por fuente de cromo y actividad de la prueba de esfuerzo.

\begin{tabular}{lccc}
\hline \multicolumn{1}{c}{ PRUEBA DE EJERCICIO } & Control & $\begin{array}{c}\text { TRATAMIENTOS } \\
\text { Levadura-Cr }\end{array}$ & Tripicolinato - $\mathbf{C r}$ \\
\hline Pre - ejercicio & 97,82 & 82,78 & 87,9 \\
Post - ejercicio 0 horas & 72,66 & 73,42 & 69,24 \\
Post - ejercicio 3 horas & 71,58 & 69,92 & 73,96 \\
Post - ejercicio 4 horas & 63,82 & 67,96 & 64,98 \\
\hline
\end{tabular}

En la siguiente tabla se indica que el análisis ANOVA no mostró diferencias entre los tratamientos $(P>0.05)$.

Tabla 3. Media de la concentración de cortisol en sangre según la fuente de cromo y la actividad de la prueba de esfuerzo.

\begin{tabular}{lccc}
\hline \multicolumn{1}{c}{ PRUEBA DE EJERCICIO } & Control & $\begin{array}{c}\text { TRATAMIENTOS } \\
\text { Levadura-Cr }\end{array}$ & Tripicolinato $-\mathbf{C r}$ \\
\hline Pre-ejercicio & 3,6 & 2,69 & 2,28 \\
Post-ejercicio 0 horas & $5,58 \mathrm{a}$ & $3,42 \mathrm{~b}$ & $2,46 \mathrm{~b}$ \\
Post-ejercicio 3 horas & 2,32 & 2,14 & 2,9 \\
Post-ejercicio 6 hoeas & 1,4 & 1,62 & 2 \\
\hline
\end{tabular}

$a=$ diferencia media significativa entre el grupo de control y los grupos de fuente de cromo.

$\mathrm{b}=$ diferencia media no significativa entre fuentes de cromo.

En la siguiente tabla el análisis ANOVA no mostró diferencias entre los tratamientos ( $P>0.05)$ antes del ejercicio y después de 3 y 6 horas.

Tabla 4. Media de la concentración de insulina en sangre por fuente de cromo y actividad de la prueba de esfuerzo.

\begin{tabular}{lccc}
\multicolumn{1}{r}{ PRUEBA DE EJERCICIO } & \multicolumn{2}{c}{ TRATAMIENTOS } \\
& Control & Levadura-Cr & Tripicolinato - Cr \\
\hline Pre ejercicio & 5,88 & 4,92 & 3,74 \\
Post-ejercicio 0 horas & $6,16 \mathrm{a}$ & $3,72 \mathrm{~b}$ \\
Post-ejercicio 3 horas & $12,14 \mathrm{a}$ & $4,45 \mathrm{~b}$ & $4,59 \mathrm{~b}$ \\
Post-ejercicio 6 horas & 3,3 & $2,94 \mathrm{~b}$ & 2,88 \\
\hline
\end{tabular}

$\mathrm{a}=$ diferencia media significativa entre el grupo de control y los grupos de fuente de cromo.

$\mathrm{b}=$ Diferencia media no significativa entre fuentes de cromo

En esta tabla el análisis ANOVA no mostró diferencias entre los tratamientos $(P>0.05)$ antes del ejercicio y después de 6 horas. Importancia entre tratamientos post-ejercicio 0 horas y 3 horas. 
a)

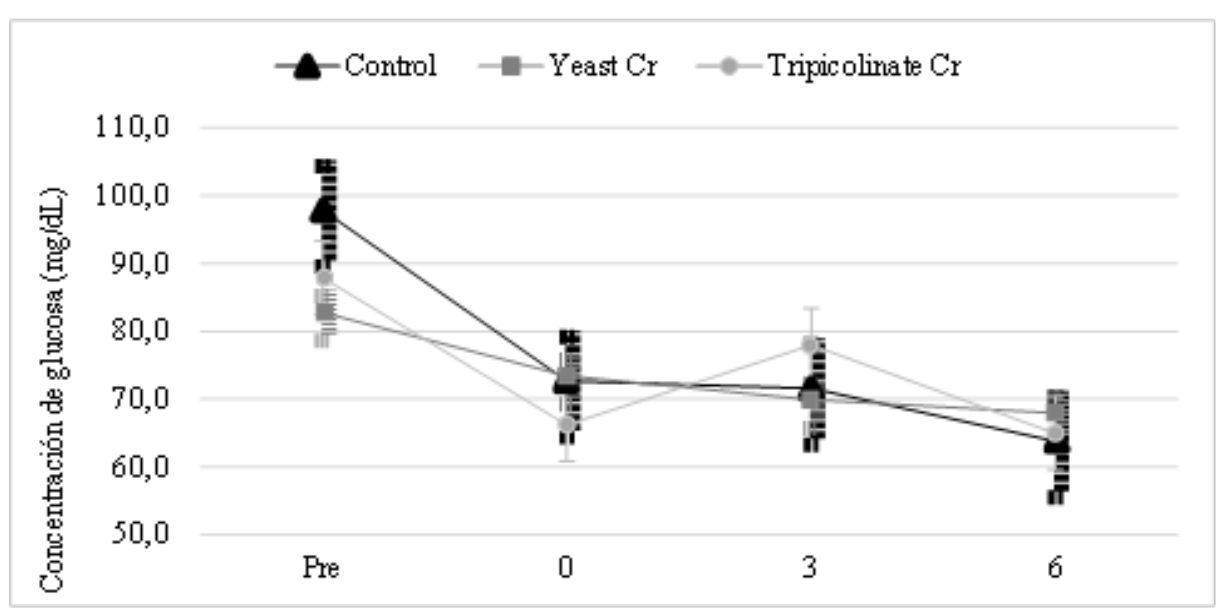

b)

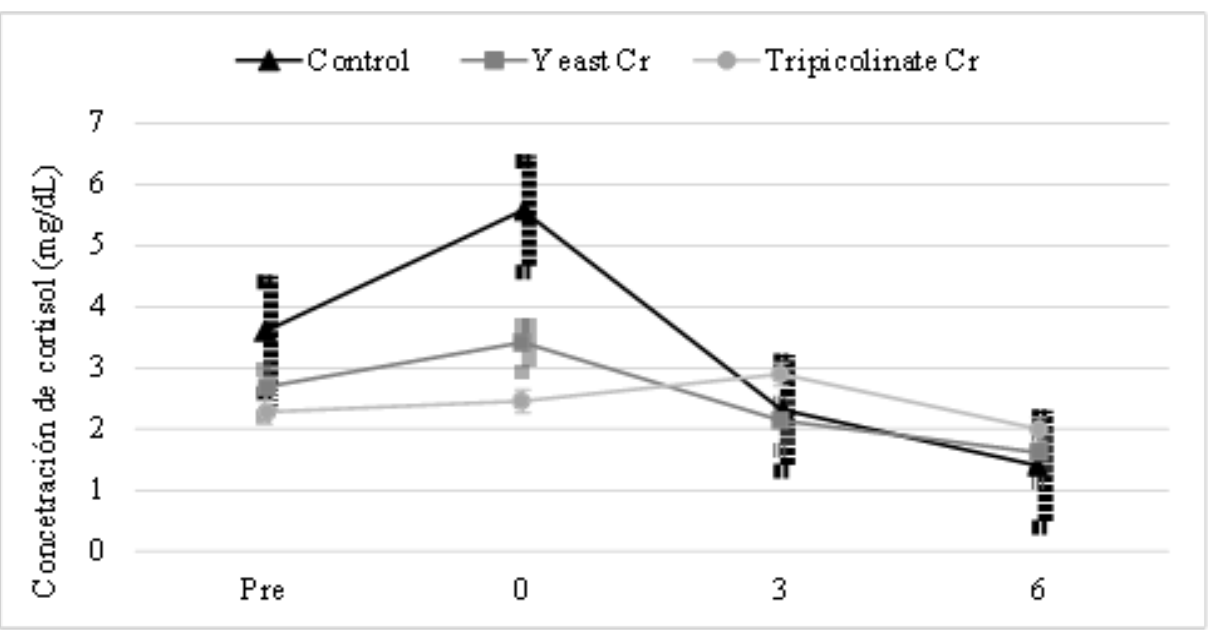

c)

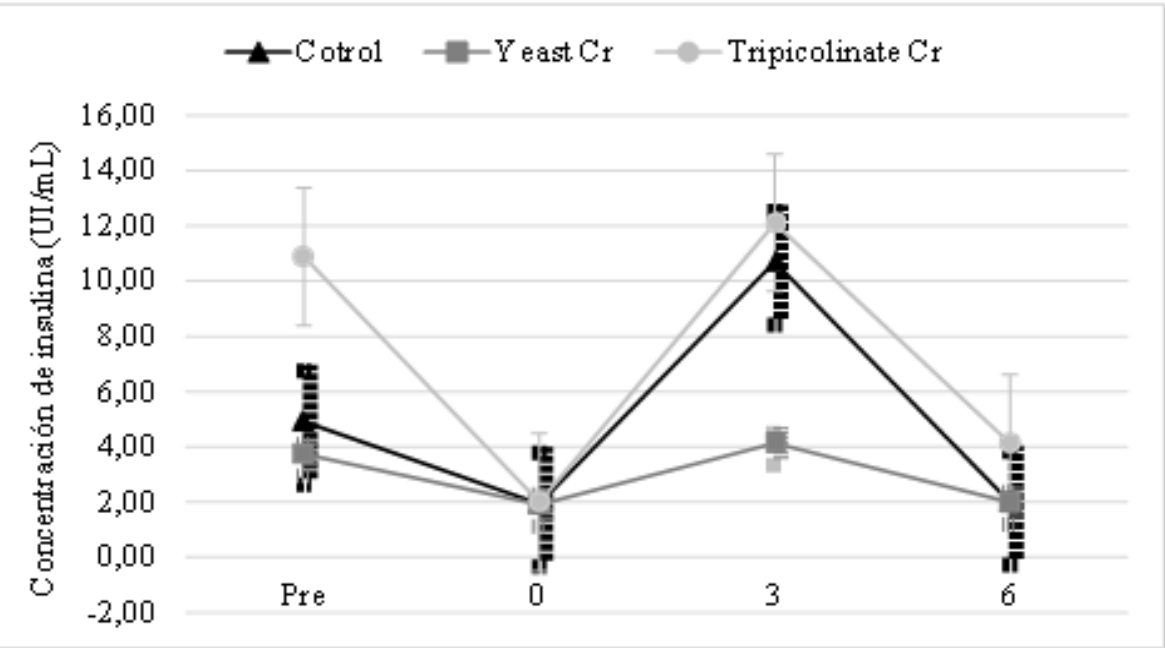

Figura 1. Media de las concentraciones sanguíneas de glucosa, cortisol e insulina en caballos deportivos bajo dos fuentes diferentes de cromo antes y después de una actividad de ejercicio intensivo. a) Niveles de glucosa, b) Niveles de cortisol y c) Niveles de insulina. 


\section{Discusión}

El análisis estadístico de los parámetros sanguíneos analizados en este estudio no reveló diferencias significativas entre los grupos de tratamiento debido a la inclusión dietética de cromo orgánico a glucosa y cortisol, excepto inmediatamente después del ejercicio (tiempo 0). Estos resultados indican que el cromo orgánico no está influenciado en la glucosa en sangre y el nivel de cortisol, excepto el cortisol inmediatamente después del ejercicio, contrariamente (19) informó que la suplementación con cromo como tripicolinato de cromo puede reducir las concentraciones de glucosa en sangre en caballos en crecimiento. El nivel plasmático máximo de glucosa se observó dos horas y media después de la ingestión (20).

La suplementación con cromo orgánico resultó en una reducción de los valores de lactato después de la actividad física, posiblemente indicando que el cromo contribuyó a una mejor utilización de la glucosa plasmática y a una mejor adaptación de los animales a la actividad física $(21,22)$. Además, el informe sugiere que tanto la actividad física como el estrés dan como resultado un aumento en el nivel de cortisol plasmático (23). El nivel de cortisol en plasma está directamente influenciado por el incidente de estrés, cuando el estrés es más intenso, el nivel de cortisol en sangre es alto. Durante la competición o el ejercicio intensivo, los caballos sufren un incidente de estrés, el estrés puede afectar a los jinetes y a los caballos $(24,25)$. Este estrés puede afectar la salud, el bienestary / o el rendimiento, porque el cortisol es ampliamente reconocido por reducir la respuesta inmune en animales y humanos (25). Sin embargo, la investigación demostró una mayor concentración de cortisol a medida que se extiende e intensifica el ejercicio y luego disminuye si se termina el ejercicio $(11,26,27)$.

Sin embargo, la concentración sérica de insulina se ve influenciada por lo tanto la suplementación con cromo y la fuente orgánica. Los niveles más altos de insulina están relacionados con la resistencia a la insulina y la laminitis en los caballos (28), donde la secreción de insulina aumenta para compensar la disminución de la respuesta del tejido a la insulina. La resistencia a la insulina puede disminuir la disponibilidad de glucosa para las células de insulina diana, porque el aumento de la secreción de insulina compensa parcialmente la respuesta disminuida de los tejidos a la insulina y se ha observado en purasangres, terneros y humanos (9,28-30), teniendo en cuenta que la insulina también es una hormona importante en la regulación del metabolismo, el crecimiento y la circulación de las grasas. Los efectos positivos pueden estar relacionados con el papel del cromo como componente integral del factor de tolerancia a la glucosa (GTF), que potencia la acción de la insulina, una de las hormonas anabólicas más importantes (31).

Además, el nivel plasmático de insulina en caballos depende de qué tipo de dieta, el caballo alimentado con una dieta a base de maíz fue estadísticamente más alto que los otros cereales a las tres horas y media después de la ingestión (32). Los caballos mostraron a ejercicios moderados, los valores de glucosa aumentaron, la insulina disminuyó y el cortisol aumentó durante la prueba y luego regresaron a los valores basales seis horas después del final del ejercicio (33). 


\section{CONCLUSIONES}

A partir del estudio anterior, se concluyó que la suplementación dietética de cromo orgánico, levadura y forma de tripicolinato, potencia el efecto de la insulina después del ejercicio y, por lo tanto, puede usarse para trabajos intensivos de caballos.

Se identificó diferencias en la suplementación de cromo en la dieta de equinos deportistas en comparación con los testigos, la suplementación dietética de cromo orgánico, en forma de tripicolinato y levadura enriquecida disminuyó los niveles de cortisol al momento de realizar actividades intensas.

El cromo suplementado en forma de levadura potenció el efecto de la insulina posterior al ejercicio, disminuyendo sus niveles en sangre a diferencia de la forma de tripicolinato y manteniéndose estable post ejercicio. Esto destaca la necesidad de realizar más investigaciones sobre la suplementación de cromo en el caballo deportista.

\section{REFERENCIAS BIBLIOGRÁFICAS}

1. Finno CJ, McKenzie E, Valberg SJ, Pagan J. Effect of fitness on glucose, insulin and cortisol responses to diets varying in starch and fat content in Thoroughbred horses with recurrent exertional rhabdomyolysis. Equine Vet J. 2010; 42(SUPPL. 38):323-8. DOI:10.1111/j.20423306.2010.00199.x

2. Bamford NJ, Potter SJ, Harris PA, Bailey SR. Breed differences in insulin sensitivity and insulinemic responses to oral glucose in horses and ponies of moderate body condition score. Domest Anim Endocrinol. 2014;47(1):101-7. DOI: 10.1016/j.domaniend.2013.11.001

3. Chameroy KA, Frank N, Elliott SB, Boston RC. Effects of a supplement containing chromium and magnesium on morphometric measurements, resting glucose, insulin concentrations and insulin sensitivity in laminitic obese horses. Equine Vet J. 2011;43(4):494-9. DOI: 10.1111/j.2042-3306.2010.00302.x

4. Pagan JD. Micromineral Requirements in Horses. En: Advances in Equine Nutrition III. Nottringham, UK; 2000. p. 317-26. http://www.mitchellplainfarm. com/uploads/2/9/4/4/2944849/pagan micromineral_requirements.pdf

5. Pagan JD, Pagan JOED, Jackson SG, Duren SE. the Effect of Chromium Supplementation on Metabolic Response to Exercise in Thoroughbred Horses. Proc Equine Nutr Symp. 1995; 96-101. https://ker.com/wpcontent/uploads/The-Effect-of-ChromiumSupplementation-on-Metabolic-Response-toExercise-in-Thoroughbred-Horses.pdf

6. Frank N, Tadros EM. Insulin dysregulation. Equine Vet J. 2014; 46(1):103-12. DOI: 10.1111/ evj.12169

7. Almeida MLM, Feringer WH, Júnior, Carvalho JRG, Rodrigues IM, Jordão LR, et al. Intense exercise and aerobic conditioning associated with chromium or L-Carnitine supplementation modified the fecal microbiota of fillies. PLoS One. 2016; 11(12):1-21. https://journals.plos. org/plosone/article?id=10.1371/journal. pone.0167108

8. Shade K-TC, Platzer B, Washburn N, Mani V, Bartsch YC, Conroy M, et al. A single glycan on IgE is indispensable for initiation of anaphylaxis. J Exp Med. 2015;212(4):457-67. DOI: 10.1084/ jem.20142182

9. Bröjer J, Lindåse S, Hedenskog J, Alvarsson K, Nostell K. Repeatability of the combined glucose-insulin tolerance test and the effect of a stressor before testing in horses of 2 breeds. J Vet Intern Med. 2013;27(6):1543-50. DOI: 10.1111/jvim.12172

10. Bertin FR, de Laat MA. The diagnosis of equine insulin dysegulation. EQUINE Vet J. 2017;49(10):3218-21. DOI: 10.1111/evj.12703

11. Morgan RA, Mcgowan TW, Mcgowan CM. Prevalenceandriskfactorsforhyperinsulinaemia 
in ponies in Queensland, Australia. Aust Vet J. 2014; 92(4):101-6. DOI: 10.1111/avj.12159

12. Harris PA, Harris RC. Nutritional ergogenic aids in the horse - uses and abuses. Conf equine Sport Med Sci. 1998; 491-508. https://www. yumpu.com/en/document/view/50834989/ nutritional-ergogenic-aids-in-the-horsekentucky-equine-research

13. Jacob SI, Geor RJ, Weber PSD, Harris PA, McCue ME. Effect of age and dietary carbohydrate profiles on glucose and insulin dynamics in horses. Equine Vet J. 2017;(10):321821. DOI: 10.1111 evj.12745

14. Kaneko JJ, Harvey JW, Bruss ML. Clinical Biochemistry of Domestic Animals. En: Clinical Biochemistry of Domestic Animals. 6.a ed. Davis, California I.; 1997. p. 661, 890-4.

15. Mowat DN, Chang X, Yang WZ. Chelated chromium for stressed feeder calves. Can J Anim Sci. 1993; 73:49-55. https://cdnsciencepub. com/doi/pdf/10.4141/cjas93-004

16. Fonseca LA, Girardi FM, Coelho CS, Barioni G, Rangel VB, Gonçalves RC. Influence of chromium supplementation on energy metabolism in horses used in policing activity. Arq Bras Med Veterinária e Zootec. 2011;63(5):1175-80. DOI: 10.2337/dc06-0996

17. Burton JL, Roberts D, Montaldi M, Novick PJ, De Camilli P. A mammalian gunaninenucleotide-releasing protein enhances function of yeast secretory protein Sec4. Nature. 1993; 361:464-7. DOI: 10.1038/361464a0

18. Chang $X$, Mallard BA, Mowat DN. Effects of chromium on health status, blood neutrophil phagocytosis and in vitro lymphocyte blastogenesis of dairy cows. Vet Immunol Immunopathol. 1996; 52:37-52. DOI: 10.1016/0165-2427(95)05539-8

19. Gobesso AA de O, Etchichury, Mariano Tosi $\mathrm{H}$. Resposta plasmática de glicose e insulina em eqüinos alimentados com diferentes fontes de amido. Brazilian J Vet Res Anim Sci. 2009;46(4):324-31. https://www.teses.usp.br/ teses/disponiveis/74/74131/tde-05052014164429/publico/ME8191732COR.pdf
20. Kedzierski W, Cywińska A, Strzelec K, Kowalik S. Changes in salivary and plasma cortisol levels in Purebred Arabian horses during race training session. J Anim Sci. 2014;(85):313. https://www. ncbi.nlm.nih.gov/pmc/articles/PMC4852239/

21. Galindo CA, Martins CB, Comide LM, de Queiroz A, Correa J. Alteraciones metabólicas durante entrenamiento en equinos de la Raza Pura Sangre Árabe. Rev Med Vet (Bogotá). 2007;(13):77-82. https://ciencia.lasalle.edu.co/ cgi/viewcontent.cgi?article $=1117$ \&context $=\mathrm{mv}$

22. Ott EA, Kivipelto J. Influence of chromium tripicolinate on growth and glucose metabolism in yearling horses. J Anim Sci. 1999; 77:3022-30. DOI: 10.2527/1999.77113022x

23. Martos N, Ayala I. Determinación de los niveles plasmáticos de cortisol en diferentes patologías de los équidos. An Vet Murcia. 2003;19:129-40. https://revistas.um.es/ analesvet/article/view/16971/16341

24. Peeters $M$, Closson $C$, Beckers JF, Vandenheede $M$. Rider and Horse Salivary Cortisol Levels During Competition and Impact on Performance. J Equine Vet Sci. 2013;(33):155-60. https://orbi.uliege.be/ bitstream/2268/156579/1/Peeters\%20et\%20 al\%202013.pdf

25. Bamford NJ, Potter SJ, Harris PA, Bailey SR. Effect of increased adiposity on insulin sensitivity and adipokine concentrations in horses and ponies fed a high fat diet, with or without a once daily high glycaemic meal. Equine Vet J. 2016;48(3):368-73. DOI: 10.1111/evj.12434

26. Evans DL, Harris RC, Snow DH. Correlation of racing performance with blood lactate and heart rate after exercise in Thoroughbred horses. EQUINE Vet J. 1993;25(5):441-5. DOI: 10.1111/j.2042-3306.1993.tb02987.x

27. Treiber $\mathrm{KH}$, Boston RC, Kronfeld DS, Staniar WB, Harris PA. Insulin resistance and compensation in Thoroughbred weanlings adapted to high-glycemic meals. J Anim Sci. 2005;83:2357-64. DOI: 10.2527/2005.83102357x 
28. Hoffman RM, Boston RC, Stefanovski D, Kronfeld DS, Harris PA. Obesity and diet affect glucose dynamics and insulin sensitivity in Thoroughbred geldings.JAnim Sci. 2003;81:233342. DOI:10.2527/2003.8192333x

29. Stanley CC, Williams CC, Jenny BF, Fernandez JM, Bateman HG, Nipper W a, et al. Effects of feeding milk replacer once versus twice daily on glucose metabolism in Holstein and Jersey calves. J Dairy Sci. 2002; 85:2335-43. DOI: 10.3168/jds.S0022-0302(02)74313-0

30. Karikoski NP, McGowan CM, Singer ER, Asplin KE, Tulamo RM, Patterson-Kane JC. Pathology of Natural Cases of Equine Endocrinopathic Laminitis Associated With Hyperinsulinemia. Vet Pathol. 2015; 52(5):94556. DOI: $10.1177 / 0300985814549212$
31. WelchS, GebhartSSP, Bergman RN, Phillips LS. Minimal Model Analysis of Intravenous Glucose Tolerance Test-Derived Insulin Sensitivity in Diabetic Subjects. J Clin Endocrinol Metab. 1990; 71(6):1508-18. https://doi.org/10.1210/ jcem-71-6-1508

32. Anderson RA. Chromium as an essentiel nutrient for humans. Regul Toxicol Pharmacol. 1997;26:35-41. DOI: 10.1006/rtph.1997.1136

33. Holdsworth ES, Neville E. Effects of extracts of high- and low-chromium brewer's yeast on metabolism of glucose by hepatocytes from rats fed on high- or low-Cr diets. $\mathrm{Br} J$ Nutr. 1990;63:623-30. DOI: https://doi.org/10.1079/ BJN19900148 